\title{
Conformal Gravity and the Alcubierre Warp Drive Metric
}

\author{
Gabriele U. Varieschi and Zily Burstein \\ Department of Physics, Loyola Marymount University, Los Angeles, CA 90045, USA
}

Correspondence should be addressed to Gabriele U. Varieschi; gvarieschi@lmu.edu

Received 7 November 2012; Accepted 24 November 2012

Academic Editors: P. P. Avelino, R. N. Henriksen, and P. A. Hughes

Copyright (c) 2013 G. U. Varieschi and Z. Burstein. This is an open access article distributed under the Creative Commons Attribution License, which permits unrestricted use, distribution, and reproduction in any medium, provided the original work is properly cited.

\begin{abstract}
We present an analysis of the classic Alcubierre metric based on conformal gravity, rather than standard general relativity. The main characteristics of the resulting warp drive remain the same as in the original study by Alcubierre, that is, effective superluminal motion is a viable outcome of the metric. We show that for particular choices of the shaping function, the Alcubierre metric in the context of conformal gravity does not violate the weak energy condition, as was the case of the original solution. In particular, the resulting warp drive does not require the use of exotic matter. Therefore, if conformal gravity is a correct extension of general relativity, superluminal motion via an Alcubierre metric might be a realistic solution, thus allowing faster-than-light interstellar travel.
\end{abstract}

\section{Introduction}

In 1994, Alcubierre introduced the so-called Warp Drive Metric (WDM), within the framework of general relativity (GR), which allows in principle for super-luminal motion, that is, faster-than-light travel [1]. This superluminal propulsion is achieved, respectively, by expanding and contracting the space time behind and in front of a spaceship, while the spacecraft is left inside a locally flat region of space time, within the so-called warp bubble.

In this way, the spaceship can travel at arbitrarily high speeds, without violating the laws of special and general relativity, or other known physical laws. Furthermore, the spacecraft and its occupants would also be at rest in flat space time, thus immune from high accelerations and unaffected by special relativistic effects, such as time dilation. Enormous tidal forces would only be present near the edge of the warp bubble, which can be made large enough to accommodate the volume occupied by the ship.

However, Alcubierre [1] was also the first to point out that this hypothetical solution of Einstein's equations of GR would violate all three standard energy conditions (weak, dominant, and strong; see [2-4] for definitions). In particular, the violation of the weak energy condition (WEC) implies that negative energy density is required to establish the Alcubierre WDM, thus making it practically impossible to achieve this type of super-luminal motion, unless large quantities of exotic matter (i.e., with negative energy density) can be created. Since our current knowledge of this type of exotic matter is limited to some special effects in quantum field theory (such as the Casimir effect), it is unlikely that the Alcubierre WDM can be practically established within the framework of general relativity.

Following Alcubierre's seminal paper, many other studies appeared in the literature, either proposing alternatives to the original warp drive mechanism $([5,6])$ or refining and analyzing in more detail the original idea ([7-19]). However, all these studies were conducted using standard GR and could not avoid the violation of the WEC, meaning that some exotic matter would always be required for faster-than-light travel. Similar issues also exist in other well-known GR solutions for super-luminal motion, such as space-time wormholes [20].

Einstein's general relativity and the related "Standard Model" of cosmology have been highly successful in describing our universe, from the solar system up to the largest cosmological scales, but recently these theories have also led to a profound crisis in our understanding of its ultimate composition. From the original discovery of the expansion of the universe, which resulted in standard big bang cosmology, scientists have progressed a long way towards our current picture, in which the contents of the universe are today described in terms of two main components, dark matter 
(DM) and dark energy (DE), accounting for most of the observed universe, with ordinary matter just playing a minor role.

Since there is no evidence available yet as to the real nature of dark matter and dark energy, alternative gravitational and cosmological theories are being developed, in addition to standard explanations of dark matter/dark energy invoking the existence of exotic new particles also yet to be discovered. In line with these possible new theoretical ideas, conformal gravity (CG) has emerged as a nonstandard extension of Einstein's GR, based on a possible symmetry of the universe: the conformal symmetry, that is, the invariability of the space-time fabric under local "stretching" of the metric (for reviews see $[21,22]$ ). This alternative theory has been reintroduced in recent years (following the original work by Weyl [23-25]), leading to cosmological models which do not require the existence of DM and DE ([26-32]).

At the quantum level conformal gravity, as well as other theories with higher derivatives, was thought to be affected by the presence of "ghosts," leading to possible instabilities of the quantum version of the theory. However, recent studies [26] have shown that CG as a quantum theory is both renormalizable and unitary, thus providing a solution to the ghost problem.

In view of a possible extension of Einstein's general relativity into conformal gravity, in this paper we have reconsidered the Alcubierre WDM, basing it on CG rather than standard GR. In Section 2, we review the fundamental principles of CG and the calculation of the stress-energy tensor in this gravitational theory. In Section 3, we consider the Alcubierre metric in CG and compute the energy density for different shaping functions of the metric.

In particular, we will show that for certain shaping functions, the Alcubierre metric in the context of conformal gravity does not violate the weak energy condition, as was the case of the original solution. This analysis continues in Section 4, where we study other energy conditions and estimate the total energy required for this CG warp drive. Finally, in Section 5, we conclude that if CG is a correct extension of GR, super-luminal motion via an Alcubierre metric might be a realistic possibility, thus enabling fasterthan-light interstellar travel without requiring exotic matter.

\section{Conformal Gravity and the Stress-Energy Tensor}

Weyl in 1918 ([23-25]) developed the "conformal" generalization of Einstein's relativity by introducing the conformal (or Weyl) tensor, a special combination of the Riemann tensor $R_{\lambda \mu \nu \kappa}$, the Ricci tensor $R_{\mu \nu}=R_{\mu \lambda \nu}^{\lambda}$, and the curvature (or Ricci) scalar $R=R_{\mu}^{\mu}$ [33] as follows:

$$
\begin{aligned}
C_{\lambda \mu \nu \kappa}= & R_{\lambda \mu \nu \kappa}-\frac{1}{2}\left(g_{\lambda \nu} R_{\mu \kappa}-g_{\lambda \kappa} R_{\mu \nu}-g_{\mu \nu} R_{\lambda \kappa}+g_{\mu \kappa} R_{\lambda \nu}\right) \\
& +\frac{1}{6} R\left(g_{\lambda \nu} g_{\mu \kappa}-g_{\lambda \kappa} g_{\mu \nu}\right)
\end{aligned}
$$

where $C_{\mu \lambda v}^{\lambda}(x)$ is invariant under the local transformation of the following metric:

$$
g_{\mu \nu}(x) \longrightarrow \widehat{g}_{\mu \nu}(x)=e^{2 \alpha(x)} g_{\mu \nu}(x)=\Omega^{2}(x) g_{\mu \nu}(x) \text {. }
$$

The factor $\Omega(x)=e^{\alpha(x)}$ determines the amount of local "stretching" of the geometry, hence the name "conformal" for a theory invariant under all local stretchings of the space-time (see [22] and references therein for more details).

This conformally invariant generalization of GR was found to be a fourth-order theory, as opposed to the standard second-order general relativity, since the field equations originating from a conformally invariant Lagrangian contain derivatives up to the fourth order of the metric, with respect to the space-time coordinates. Following the works done by Bach [34], Lanczos [35], and others, CG was ultimately based on the following Weyl or conformal action (In this paper we adopt a metric signature $(-,+,+,+)$ and we follow the sign conventions of Weinberg [33]. In this section we will leave fundamental constants, such as $c$ and $G$, in all equations, but later we will use geometrized units ( $c=1, G=1)$, or c.g.s. units when needed.):

$$
I_{W}=-\alpha_{g} \int d^{4} x(-g)^{1 / 2} C_{\lambda \mu \nu \kappa} C^{\lambda \mu \nu \kappa},
$$

or on the following equivalent expression, differing from the previous one only by a topological invariant:

$$
I_{W}=-2 \alpha_{g} \int d^{4} x(-g)^{1 / 2}\left(R_{\mu \kappa} R^{\mu \kappa}-\frac{1}{3} R^{2}\right),
$$

where $g \equiv \operatorname{det}\left(g_{\mu \nu}\right)$ and $\alpha_{g}$ is the gravitational coupling constant of conformal gravity (see [21, 36-38].) (In these cited papers, $\alpha_{g}$ is considered a dimensionless constant by using natural units. Working with c.g.s. units, we can assign dimensions of an action to the constant $\alpha_{g}$ so that the dimensionality of (5) will be correct.) Under the conformal transformation in (2), the Weyl tensor transforms as $C_{\lambda \mu \nu \kappa} \rightarrow \widehat{C_{\lambda \mu \nu \kappa}}=$ $e^{2 \alpha(x)} C_{\lambda \mu \nu \kappa}=\Omega^{2}(x) C_{\lambda \mu \nu \kappa}$, while the conformal action $I_{W}$ is locally conformally invariant, the only general coordinate scalar action with such properties.

Bach [34] introduced the gravitational field equations in the presence of a stress-energy tensor (We follow here the convention [21] of introducing the stress-energy tensor $T_{\mu \nu}$ so that the quantity $c T_{00}$ has the dimensions of an energy density.) $T_{\mu \nu}$ as

$$
W_{\mu \nu}=\frac{1}{4 \alpha_{g}} T_{\mu \nu}
$$

as opposed to Einstein's standard equations as

$$
G_{\mu \nu}=R_{\mu \nu}-\frac{1}{2} g_{\mu \nu} R=-\frac{8 \pi G}{c^{3}} T_{\mu \nu}
$$

where the "Bach tensor" $W_{\mu \nu}$ [34] is the equivalent in CG of the Einstein curvature tensor $G_{\mu \nu}$ on the left-hand side of (6). 
$W_{\mu \nu}$ has a very complex structure and can be defined in a compact way as [39]

$$
W_{\mu \nu}=2 C_{\mu \nu ; \beta ; \alpha}^{\alpha}+C_{\mu \nu}^{\alpha}{ }^{\beta} R_{\beta \alpha},
$$

or in an expanded form as $([37,40])$

$$
\begin{aligned}
W_{\mu \nu}= & -\frac{1}{6} g_{\mu \nu} R_{; \lambda}^{; \lambda}+\frac{2}{3} R_{; \mu ; \nu}+R_{\mu \nu}^{; \lambda} ; \lambda \\
& -R_{\mu}{ }^{\lambda}{ }_{; \nu ; \lambda}-R_{\nu ; \mu ; \lambda}{ }^{\lambda}+\frac{2}{3} R R_{\mu \nu} \\
& -2 R_{\mu}{ }^{\lambda} R_{\lambda \nu}+\frac{1}{2} g_{\mu \nu} R_{\lambda \rho} R^{\lambda \rho}-\frac{1}{6} g_{\mu \nu} R^{2},
\end{aligned}
$$

involving derivatives up to the fourth order of the metric with respect to space-time coordinates.

Therefore, in conformal gravity, the stress-energy tensor is computed by combining together (5) and (8) as

$$
\begin{aligned}
T_{\mu \nu}=4 \alpha_{g} W_{\mu \nu}=4 \alpha_{g}( & -\frac{1}{6} g_{\mu \nu} R_{; \lambda}^{; \lambda}+\frac{2}{3} R_{; \mu ; \nu}+R_{\mu \nu ; \lambda}^{; \lambda} \\
& -R_{\mu}{ }^{\lambda} ; \nu ; \lambda \\
& -2 R_{\nu ; \mu ; \lambda}{ }^{\lambda}{ }^{\lambda} R_{\lambda \nu}+\frac{2}{3} R R_{\mu \nu} g_{\mu \nu} R_{\lambda \rho} R^{\lambda \rho} \\
& \left.-\frac{1}{6} g_{\mu \nu} R^{2}\right) .
\end{aligned}
$$

This form of the tensor will be used in the following sections, in connection to the Alcubierre metric, to compute the energy density and other relevant quantities.

For this purpose, we have developed a special Mathematica program which enables us to compute all the tensor quantities of both GR and CG, for any given metric. In particular, this program can compute the conformal tensor $C_{\lambda \mu \nu \kappa}$ in (1), the Bach tensor $W_{\mu \nu}$ in (8), or the stress-energy tensor $T_{\mu \nu}$ in (9) by performing all the necessary covariant derivatives. Given the complexity of these types of computations, we have tested extensively our program against the results for $C_{\lambda \mu \nu \kappa}$ and $W_{\mu \nu}$ computed by Kazanas and Mannheim in [38] for different metrics, obtaining a perfect agreement.

\section{Alcubierre Metric, Shaping Functions, and the Weak Energy Condition}

The original Alcubierre metric [1] considered a spaceship traveling along the $x$-axis, with motion described by a function $x_{s}(t)$ and spaceship velocity $v_{s}(t)=d x_{s}(t) / d t$. Using the $3+1$ formalism of GR, the metric was written in Cartesian coordinates as $(c=1)$

$$
d s^{2}=-d t^{2}+\left[d x-v_{s}(t) f\left(r_{s}\right) d t\right]^{2}+d y^{2}+d z^{2},
$$

where $r_{s}$ is the distance from the spaceship position as

$$
r_{s}(t)=\sqrt{\left[x-x_{s}(t)\right]^{2}+y^{2}+z^{2}}
$$

and $f\left(r_{s}\right)$ is a "form function" or "shaping function" which needs to have values $f=1$ and $f=0$, respectively, inside and outside the warp bubble, while it can have an arbitrary shape in the transition region of the warp bubble itself.

The original shaping function used by Alcubierre was

$$
f\left(r_{s}\right)=\frac{\tanh \left[\sigma\left(r_{s}+R\right)\right]-\tanh \left[\sigma\left(r_{s}-R\right)\right]}{2 \tanh (\sigma R)},
$$

where $R>0$ basically indicates the radius of the spherical warp bubble, while $\sigma>0$ relates to the bubble thickness, which decreases with increasing values of $\sigma$. In the following, we will refer to the function in (12) as the "Alcubierre shaping function" (ASF).

We will show that the particular form of the shaping function can play an important role in the energy conditions for the WDM. In our analysis we tested several different functions obeying the general requirements for $f$ outlined above. In addition to the Alcubierre function above, in this paper we will also use the following:

$$
f\left(r_{s}\right)= \begin{cases}1-\left(\frac{r_{s}}{R}\right)^{m} ; & r_{s}<R \\ 0 ; & r_{s}>R\end{cases}
$$

where $m$ is a positive integer. Since this particular function for $m=4$ is used by Hartle to illustrate the warp drive in his textbook [20], we will refer to the function in (13) as the "Hartle shaping function" (HSF).

The top panels in Figure 1 illustrate the differences between the Alcubierre shaping function (top left panel, for $\sigma=8$ as used originally by Alcubierre) and the Hartle shaping function (top right panel, for $m=4$ ). All functions in this figure are computed for a fixed value of $R=1$ and at time $t=$ 0 , when the spaceship is located at the origin. All quantities shown in the different panels are plotted as a function of the $x$ coordinate of the spaceship motion and of the transverse cylindrical coordinate $\rho=\sqrt{y^{2}+z^{2}}$. Similar coordinates will also be used in the other figures. (The cylindrical coordinate $\rho=\sqrt{y^{2}+z^{2}}$ should be considered as non-negative and all quantities in the figures plotted only for $\rho \geq 0$. However, for illustrative purposes and also to follow similar figures in the literature (such as those in $[1,11,12]$ ), we decided to let $\rho$ run on negative values in all figures, except in the last one, where we restrict $\rho \geq 0$ for a correct energy calculation.)

The expansion/contraction function $\theta$ of the volume elements behind/in front of the spaceship was also computed by Alcubierre as [1]

$$
\theta=v_{s}(t) \frac{\left[x-x_{s}(t)\right]}{r_{s}} \frac{d f\left(r_{s}\right)}{d r_{s}}
$$

and is illustrated for $v_{s}=1$ (in geometrized units, i.e., $v_{s}=c$ in traditional units) in Figure 1(b), for the two different shaping functions. Again, the choice of the parameters $R$, $\sigma$, and $m$ is the same as in the top panels in the figure. The expansion $\theta$ for the ASF is the same as Figure 1 in [1], while the corresponding $\theta$ for the HSF is slightly different but still 

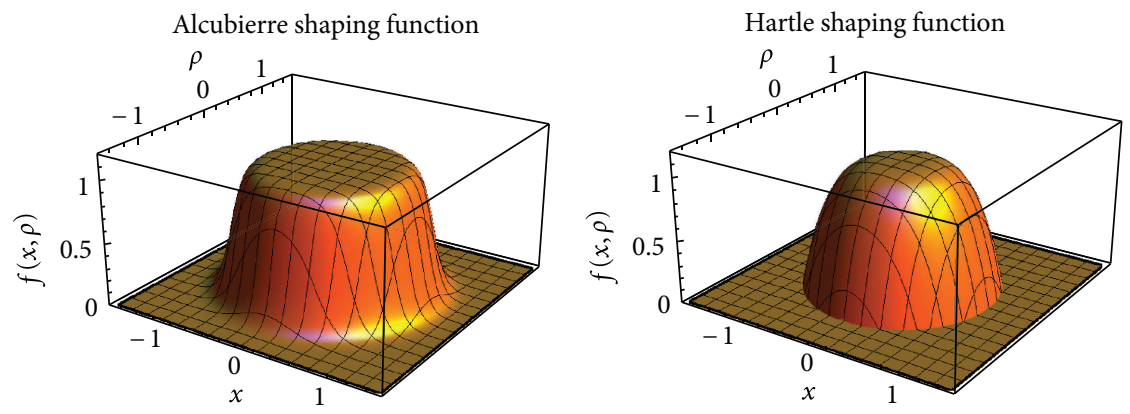

(a)
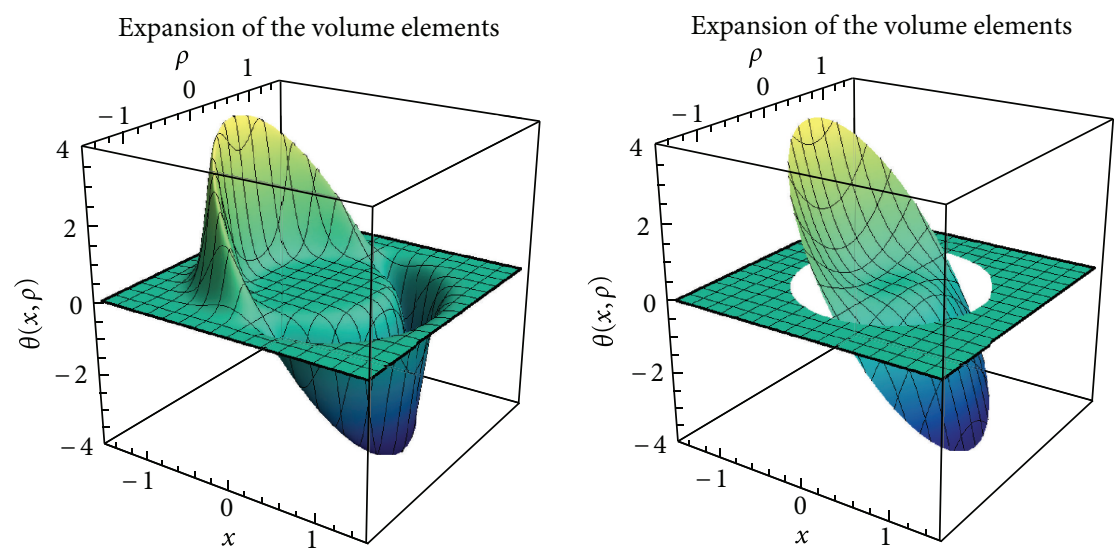

(b)
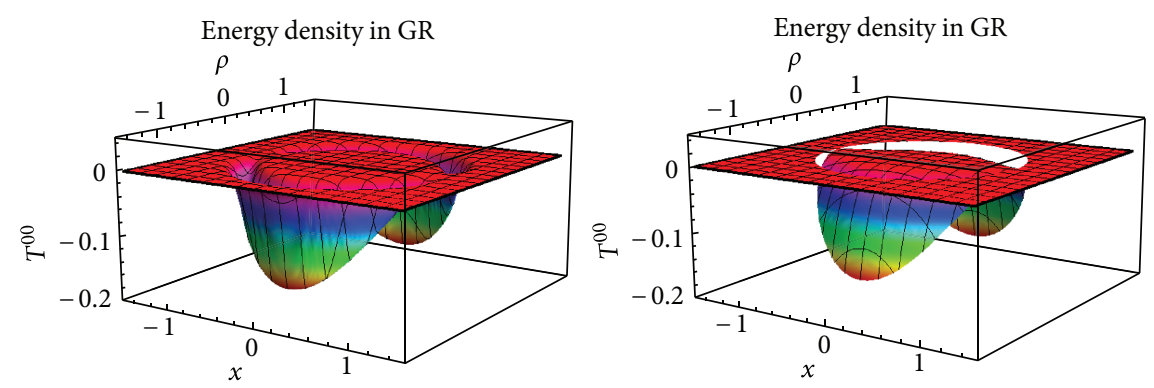

(c)
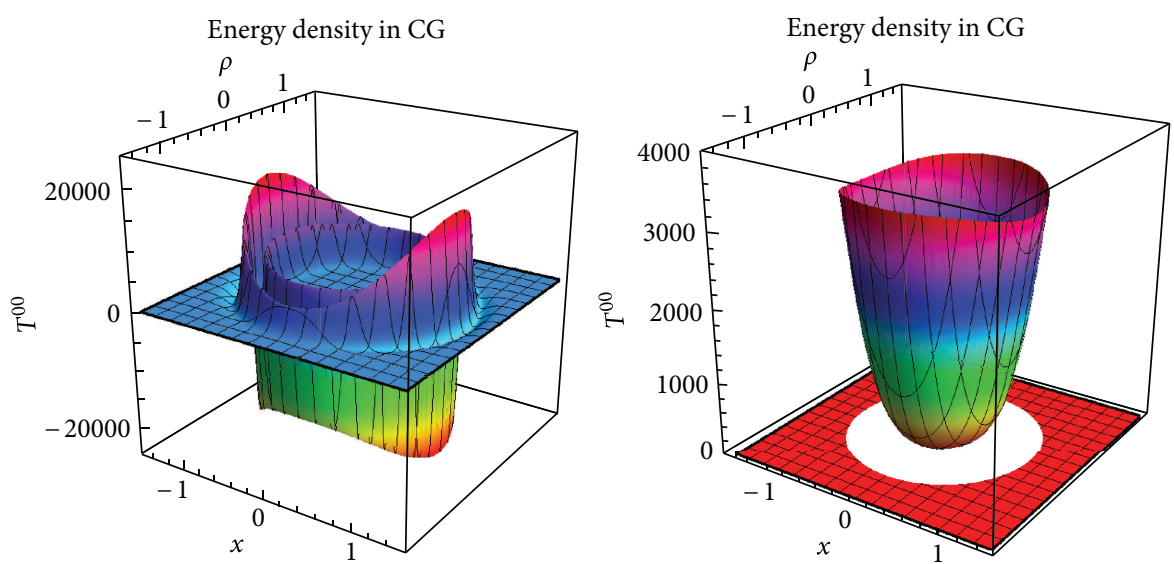

(d)

FIGURE 1: Results for the two different shaping functions (left column ASF, right column HSF), computed with parameters $v_{s}=1, R=1$, $\sigma=8, m=4, \alpha_{g}=1, t=0$. (a) Alcubierre and Hartle shaping functions. (b) Expansion of the volume elements $\theta$. (c) Energy density $T^{00}$ computed with general relativity. (d) Energy density $T^{00}$ computed with conformal gravity. The WEC is verified in the case shown in the bottom right panel. 
shows expansion of the normal volume elements behind the spaceship and contraction in front of it.

The weak energy condition $([2-4])$ requires that $T_{\mu \nu} t^{\mu} t^{\nu} \geq 0$ for all timelike vectors $t^{\mu}$. Alcubierre has also shown that for the Eulerian observers in the warp drive metric, and for their 4 -velocity $n_{\mu}$, the following relation holds [1]:

$$
T^{\mu \nu} n_{\mu} n_{v}=T^{00}=-\frac{1}{8 \pi} \frac{v_{s}^{2} \rho^{2}}{4 r_{s}^{2}}\left(\frac{d f}{d r_{s}}\right)^{2},
$$

which implies that the energy density $T^{00}$ is negative everywhere for any choice of the shaping function $f$ and, therefore, the WEC is violated (also the dominant energy condition, DEC, and the strong energy condition, SEC, are violated in the analysis based on GR [1]).

This violation of the WEC in GR is illustrated in Figure 1(c), where $T^{00}$ is calculated using (15) for both shaping functions. Although the results in the two panels are slightly different, they obviously show negative energy densities and therefore a complete violation of the WEC.

The situation is different if we compute the energy density $T^{00}$ in the framework of CG, following (9), setting $\alpha_{g}=1$ for simplicity, and using the completely contravariant form of the stress-energy tensor, instead of the covariant one. As seen in the bottom row of Figure 1, the energy density in CG is completely different from the one calculated within the framework of GR. In the bottom left panel $T^{00}$ is computed with the ASF and the resulting function is in part positive and in part negative, thus still violating the WEC.

However, the bottom right panel shows $T^{00}$ computed with the HSF and in this case the energy density is completely non-negative, showing that the WEC is verified and no exotic matter is needed to establish the warp drive. (In this panel of Figure 1 (and also in the other figures) we show a step discontinuity at the edge of the warp bubble, common to all our CG solutions. These discontinuities can be mathematically replaced by appropriate delta functions, as described by the equations used in the Appendix to model the Hartle shaping function. However, these delta functions do not need to be included in the physical solutions for $T^{00}$ in CG shown in the figures. Therefore, these energy densities are completely non-negative and they fully determine the expansion of the volume elements required for the warp drive effect.) This non-negative energy density plot in the bottom right panel of Figure 1 is the main result of our paper as it shows that-if CG is the correct extension of GR-it might be possible to establish a warp drive without having to use negative energy (mass), thus overcoming the main difficulty of the warp drive mechanism.

The explicit expression of $T^{00}$ in CG, computed with our Mathematica program, is rather cumbersome and is reproduced in (A.1) of the Appendix. Here we present just the graphical computation of $T^{00}$ in Figure 1, or in the other figures in this paper. We have tested the validity of these results by running the program in several different ways, including computing the stress-energy tensor for a simplified three-dimensional Alcubierre metric (coordinates $x, y$, and $t$ only), always obtaining consistent results. The differences in the two plots at the bottom of the figure can be attributed to the different shaping functions and their derivatives up to the fourth order. All these derivatives enter the complex expression of $T^{\mu \nu}$ in CG, as in the master equation (9), and their interplay ultimately determines the shape of $T^{00}$, or of the other components, in a way which is hard to predict before the actual computation is performed.

In Figure 2, we analyze the dependence of the energy density $T^{00}$ on the spaceship velocity $v_{s}$. In this case we consider only the Hartle shaping function for $m=4$ and $R=1$, and we compute $T^{00}$ in CG $\left(\alpha_{g}=1\right)$ for speeds ranging from the subluminal $v_{s}=0.25 c$ to the super-luminal $v_{s}=3.00 \mathrm{c}$ (for $v_{s}=1.00 \mathrm{c}$ we obtain the same function as in the bottom right panel of Figure 1). The shape of the energy density function is about the same for speeds up to $v_{s}=1.50 c$, although the function values increase with speed. For higher velocities, the function develops two "downward lobes" which eventually become negative for speeds $v_{s} \gtrsim$ $2.50 c$. This implies that the WEC is verified for speeds up to $v_{s} \approx 2.50 c$, while at higher velocities exotic matter would be required to sustain the warp drive.

This apparent "speed limit" at about $v_{s} \approx 2.50 \mathrm{c}$ might be raised or overcome completely by adopting a different shaping function, instead of the HSF used here, but this analysis would go beyond the scope of this work. We do not ascribe any physical significance to this new speed limit, which is probably just an artificial product of the shaping functions used in this analysis.

In any case, the results reported in Figure 2 show that a warp drive in CG with positive energy density is possible for a wide range of spaceship velocities; therefore, if CG is the correct extension of GR, the Alcubierre warp drive might be a viable mechanism for super-luminal travel.

In Figure 3, we present the other components of the stress-energy tensor. These were computed with the same Mathematica program, following (9) with $\alpha_{g}=1$, leading to even more complex expressions than the one for $T^{00}$ (we will omit to report these expressions for brevity). To simplify the computation, we used the covariant components $T_{\mu \nu}$ and adopted cylindrical coordinates around the $x$-axis, $(t, x, \rho, \phi) \equiv(0,1,2,3)$, instead of the Cartesian coordinates of the original Alcubierre metric. The results in the different panels are labeled accordingly. We recall that the stressenergy tensor is symmetric, $T_{\mu \nu}=T_{\nu \mu}$, and only the nonzero components are illustrated in this figure, under similar conditions used before (CG with HSF and parameters $m=4$, $R=1, v_{s}=1.00 c$ ).

The shapes of the other components of $T^{\mu \nu}$ are more complex than the one of $T^{00}$, but they can all be determined analytically, either in covariant or contravariant form, using our program. If this exact form of the stress-energy tensor could be established in the region surrounding the spacecraft, warp drive motion would be feasible within the framework of conformal gravity.

We also want to point out that we set the spaceship motion in the positive direction of the $x$-axis (setting $v_{s}=+1.00 \mathrm{c}$ ), and this is reflected in the symmetry, or lack thereof, of the components of $T_{\mu \nu}$. While some components, such as $T^{00}$ 

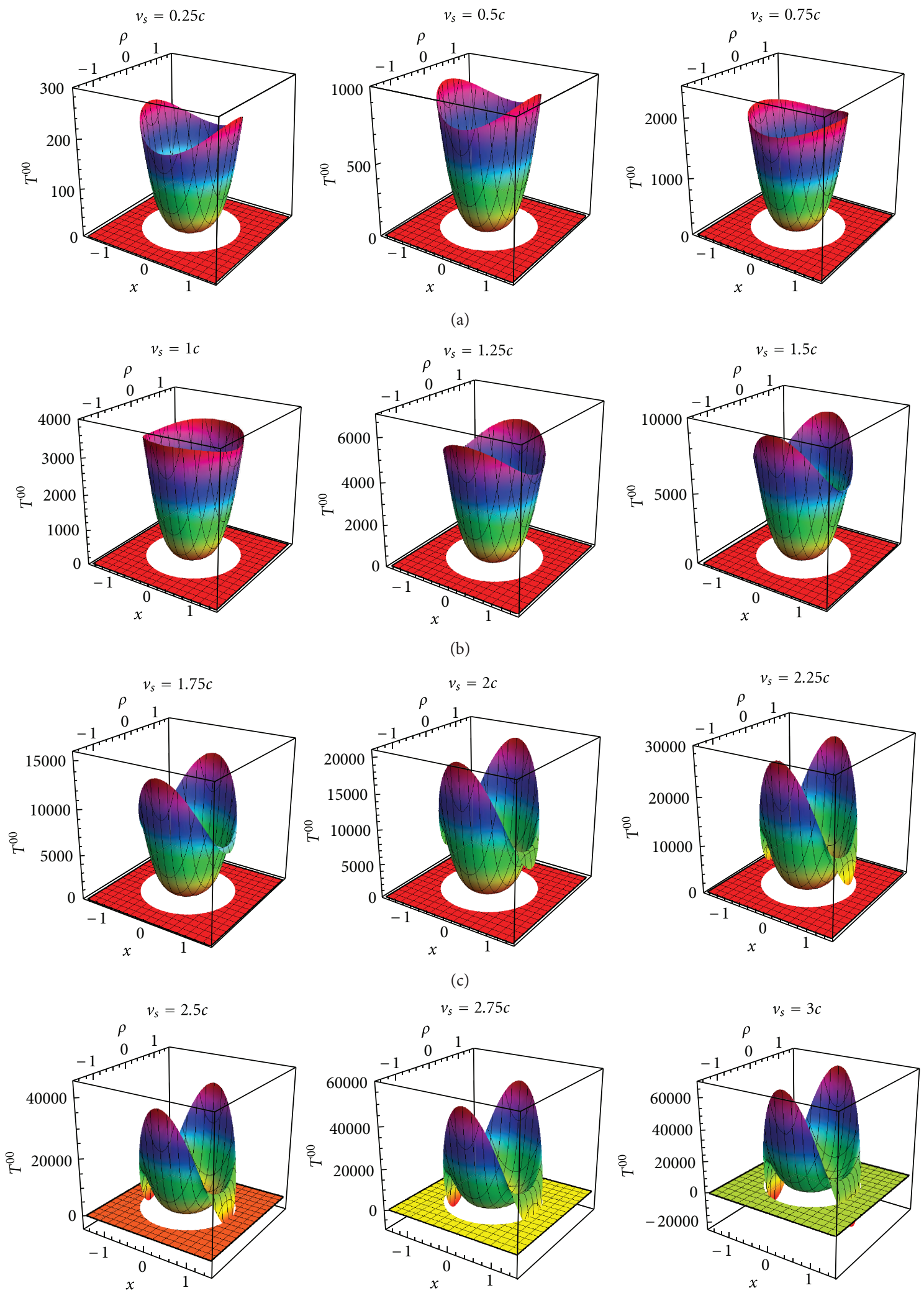

(d)

FIGURE 2: Energy density $T^{00}$ computed with conformal gravity and the Hartle shaping function. Parameters used are $R=1, m=4, \alpha_{g}=1$, $t=0$, and variable $v_{s}=0.25 c-3.00 c$. The energy density becomes in part negative for $v_{s} \gtrsim 2.50 c$, so the WEC is verified for speeds up to about $v_{s} \simeq 2.50$ c. 

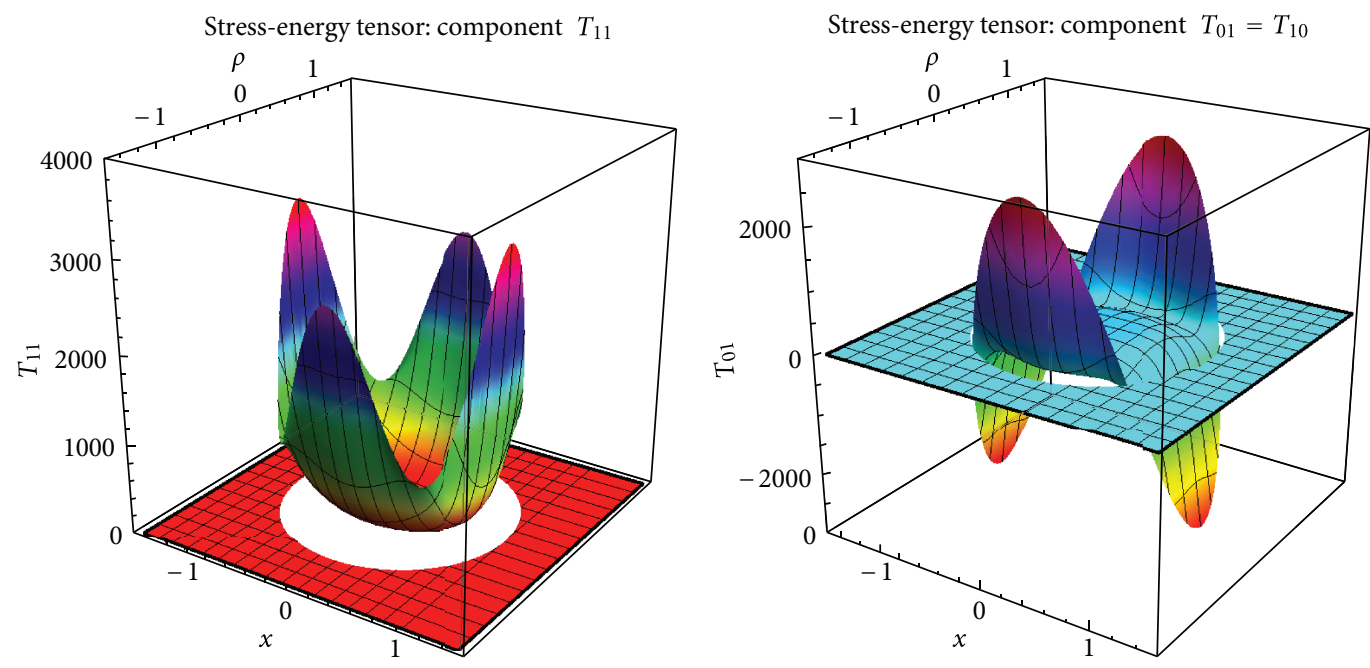

(a)
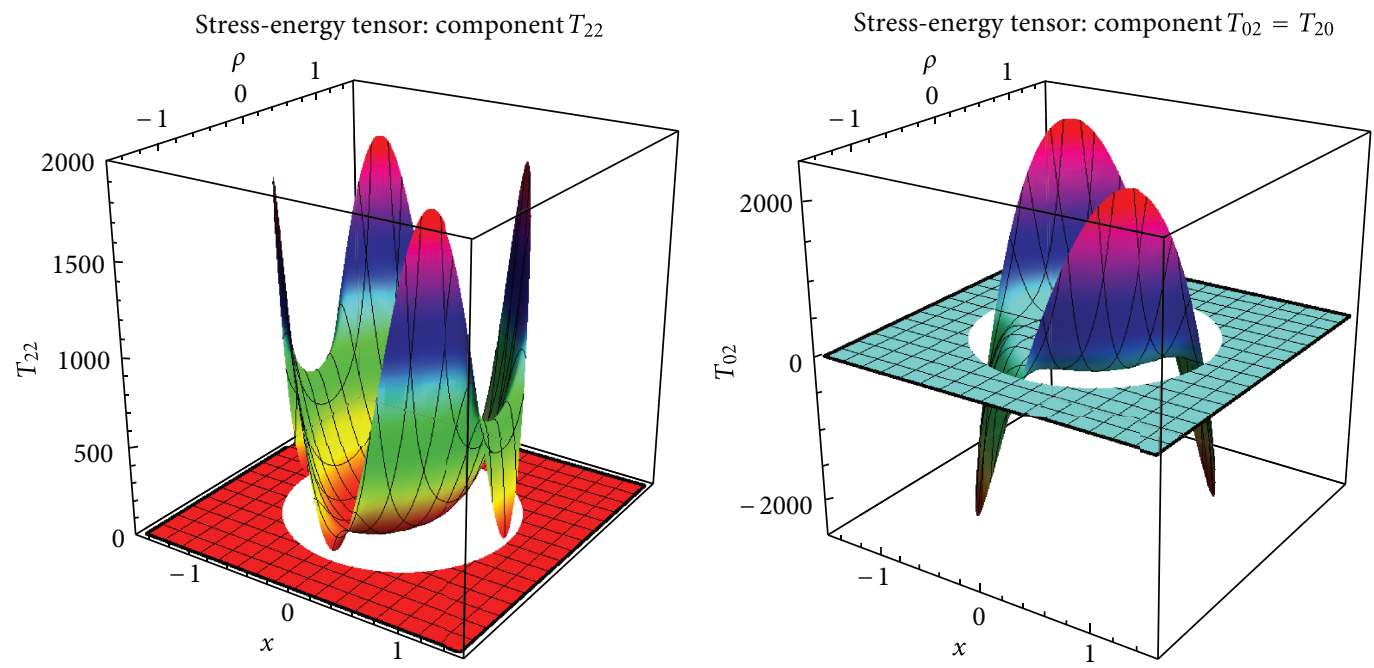

(b)
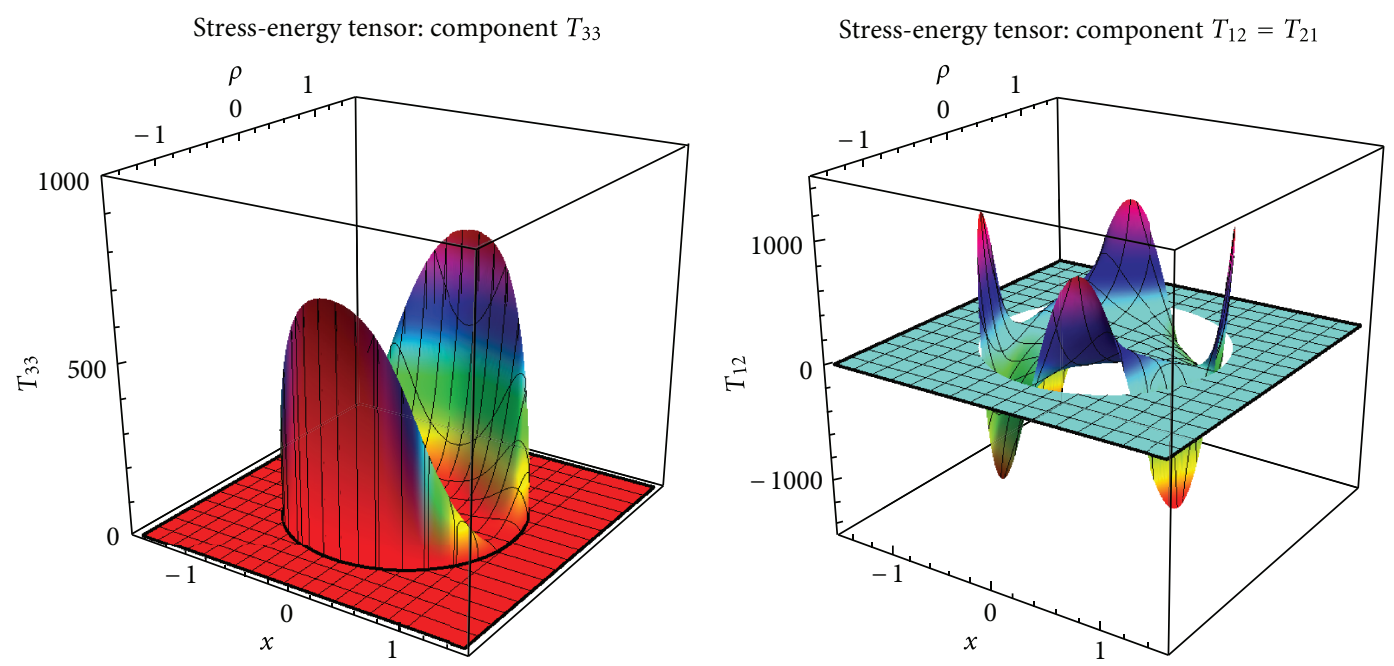

(c)

FIGURE 3: Stress-energy tensor components $T_{\mu \nu}$, in cylindrical coordinates $(t, x, \rho, \phi) \equiv(0,1,2,3)$, computed with conformal gravity and the Hartle shaping function. Parameters used are $v_{s}=1, R=1, m=4, \alpha_{g}=1$, and $t=0$. 
(or $T_{00}$ ), $T_{01}=T_{10}, T_{11}, T_{22}, T_{33}$, appear to be symmetric under the exchange $x \rightarrow-x$, the other components, $T_{02}=$ $T_{20}$ and $T_{12}=T_{21}$, are not symmetric under this exchange and, therefore, these components must contain information about the spaceship direction of motion. This argument overcomes the objection addressed in [12] that since $T^{00}$ is symmetric about the $x_{s}=0$ plane, there is uncertainty in where the space-time is expanded/contracted, thus making it impossible for the spaceship to know in which direction of the $x$-axis, positive or negative, to move. Rather, Figure 3 shows that a "bias" towards one of the two possible directions is induced by some components of $T_{\mu \nu}$.

Figure 4 illustrates one last dependence of the energy density $T^{00}$ on the parameters used. In this case, we set $R=1$, $v_{s}=1.00 \mathrm{c}$ and consider the Hartle shaping function as in (13), while varying the integer parameter $m$. In addition to our standard value, $m=4$, we have also tried values from $m=2$ to $m=10$, as shown in the figure.

In general, increasing the $m$ value increases the internal volume of the warp bubble, where space time is flat, and therefore decreases the thickness of the bubble wall where the space-time distortion takes place, in a way similar to that of the $\sigma$ parameter in the original Alcubierre warp drive. However, increasing $m$ also increases the energy required to establish the warp drive (as we checked by integrating the functions in Figure 4). Therefore, it appears to be convenient to use a low value for this parameter. As shown in the different panels of the figure, the first value, $m=2$, does not work since it does not leave a flat space-time volume inside the bubble, while $m=3$ seems to create a very small volume inside the bubble. Therefore, intermediate values such as $m=$ 4-6 would appear to be more adequate to establish the warp drive. Meanwhile, the solution for $m=10$ would require much more energy and would not give any advantage, except reducing the thickness of the warp bubble. We also checked that changing the value of $m$ does not have a strong effect on the "speed limit" of $v_{s} \approx 2.50 c$, reported above for the case $m=4$. Thus, this value of the parameter seems to be the most adequate for this type of solutions.

\section{Other Energy Conditions and Warp Drive Energy Estimate}

In the previous section, we have discussed at length the weak energy condition (WEC) for the conformal gravity Alcubierre warp drive. We have seen that, if the Hartle shaping function is used, this condition is not violated for a wide range of spaceship velocities, including super-luminal speeds. In this section, we will briefly analyze the other main energy conditions and estimate the energy necessary to establish the warp drive in CG.

The dominant energy condition (DEC) is reported in the literature ([2-4]) as $T^{00} \geq\left|T^{\mu v}\right|$ for any $\mu, v$, or equivalently as assuming the WEC plus the additional condition that $T^{\mu \nu} t_{\mu}$ is a nonspacelike vector, that is, $T_{\mu \nu} T^{\nu}{ }_{\lambda} t^{\mu} t^{\lambda} \leq 0$. It is easy to see that using as a vector $t_{\mu}$ the 4 -velocity $n_{\mu}$ of the Eulerian observers [1], the previous condition for the DEC becomes $T^{0}{ }_{\lambda} T^{0 \lambda} \leq 0$.

Figure 5 illustrates the violation of the DEC for our standard solution (AWD with HSF and $m=4, R=1$, $v_{s}=1.00 \mathrm{c}$ ). The plotted function $T^{0}{ }_{\lambda} T^{0 \lambda}$ is not negative everywhere, as required by the DEC, but shows a violation for the central portion of the warp bubble. Even if this energy condition appears to be violated, this does not notably affect the feasibility of our CG warp drive. We recall that the DEC is usually related to the standard perfect fluid stress-energy tensor, $T_{\mu \nu}=(\rho+p) U_{\mu} U_{v}+p g_{\mu v}$, where here $\rho$ and $p$ are the fluid density and pressure, while $U_{\mu}$ is the fluid 4-velocity. In this context, the DEC requires $\rho \geq|p|$, but this condition is not required in general by all classical forms of matter [3]; therefore, its violation in our case is not particularly significant.

On the contrary, our standard solution also verifies the strong energy condition (SEC) which states that $T_{\mu \nu} t^{\mu} t^{\nu} \geq$ $(1 / 2) T^{\lambda}{ }_{\lambda} t^{\sigma} t_{\sigma}$ for all timelike vectors $t^{\mu}$. Again, using the Eulerian 4-velocity vector in place of $t^{\mu}$, the previous condition is equivalent to $T^{00}+(1 / 2) T_{\lambda}^{\lambda} \geq 0$. Since the scalar $T_{\lambda}^{\lambda}$ is identically zero for all our solutions, as checked using our Mathematica program, the SEC is equivalent to $T^{00} \geq 0$, which is the WEC already verified in Section 3.

Finally, we want to estimate the energy necessary to establish our CG warp drive, under reasonable conditions. For this purpose, in Figure 6 we computed once again the energy density $T^{00}$ for our AWD with the Hartle shaping function $\left(\alpha_{g}=1, m=4, v_{s}=1.00 c\right)$, but this time for $R=10000 \mathrm{~cm}=100 \mathrm{~m}$, a reasonable radius for a warp bubble enclosing our spaceship.

Figure 6 illustrates this solution, plotted only for $\rho=$ $\sqrt{y^{2}+z^{2}} \geq 0$, as this is the correct interval for the transverse coordinate $\rho$. The cylindrical symmetry of this solution can also be better appreciated in this type of plot. We then followed the procedure outlined in $[8,11]$, to integrate the local energy density over the proper volume, in cylindrical coordinates at time $t=0$ over all space, obtaining the total energy $E$ as

$$
E=\alpha_{g} c \int d^{3} x \sqrt{|g|} T^{00}=\left(1.86 \times 10^{10} \alpha_{g}\right) \mathrm{erg},
$$

where $g=\operatorname{Det}\left|g_{i j}\right|$ is the determinant of the spatial metric on the constant time hypersurface. Since we assume that the spaceship is traveling at constant velocity, $v_{s}=1.00 c$, the total energy is also constant with time. In the last equation, we reinstated a factor of $c$ to obtain the correct dimensions (see Section 2) and also inserted an overall multiplicative factor $\alpha_{g}$, which corresponds to the conformal gravity coupling constant in (9). This factor is necessary since our computation of $T^{00}$ in Figure 6 was done assuming $\alpha_{g}=1$.

Therefore, we need to know the CG value for $\alpha_{g}$ in order to complete our energy estimation. Unfortunately, the value of this coupling constant is not well determined yet. The only value in the literature is reported by Mannheim [30] as

$$
\alpha_{g}=3.29 \times 10^{82} \mathrm{erg} \mathrm{s},
$$



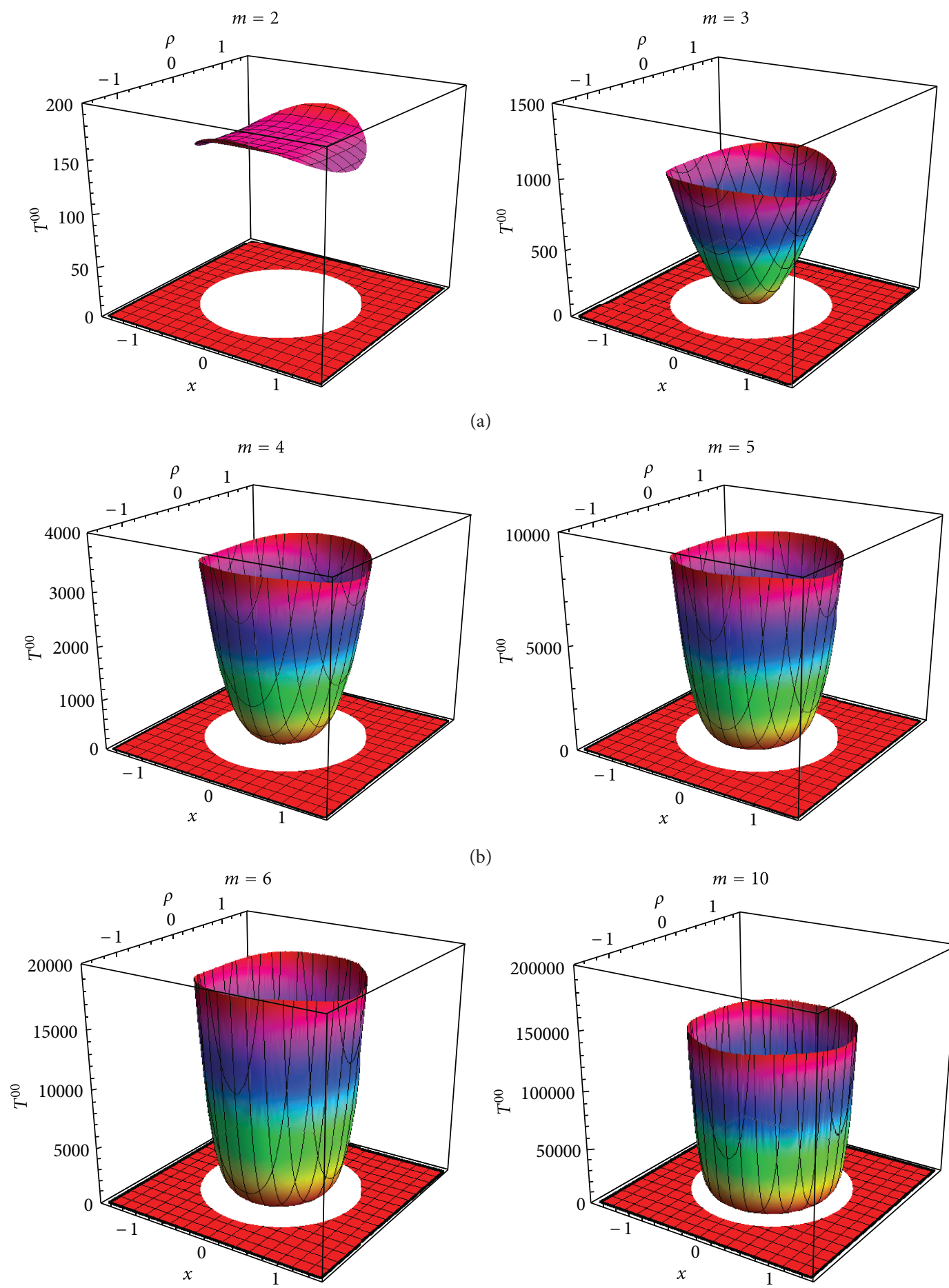

(b)

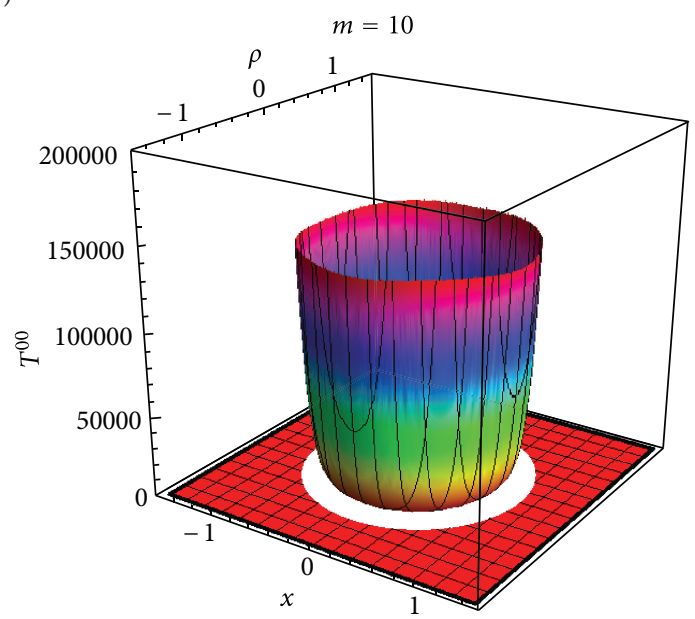

(c)

Figure 4: Energy density $T^{00}$ computed with conformal gravity and the Hartle shaping function. Parameters used are $v_{s}=1, R=1, \alpha_{g}=1$, $t=0$, and variable $m=2-10$. In all cases, the WEC is verified.

since this coupling constant has the dimensions of action. Inserting this value for $\alpha_{g}$ in (16), we obtain the energy estimate as

$$
\begin{aligned}
E & =6.12 \times 10^{92} \mathrm{erg}=6.81 \times 10^{71} \mathrm{~g} \\
& =3.42 \times 10^{38} M_{\odot},
\end{aligned}
$$

where we also converted this energy into equivalent mass and compared our result with the solar mass $M_{\odot}=1.99 \times 10^{33} \mathrm{~g}$.

The estimate in (18) would imply that an enormous amount of (standard) mass or energy is needed to establish our warp drive at a velocity equal to the speed of light, with a reasonable size for the warp bubble. However, the CG 


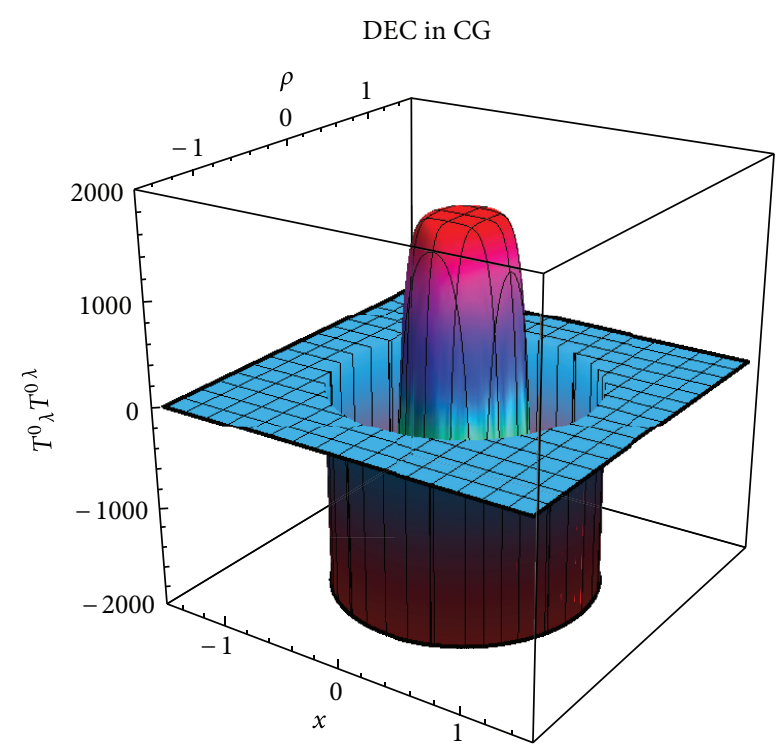

FIGURE 5: Violation of the DEC in the case analyzed (CG with HSF and $\left.m=4, R=1, v_{s}=1.00 c, \alpha_{g}=1, t=0\right)$. The plotted function $T^{0}{ }_{\lambda} T^{0 \lambda}$ is not negative everywhere, as required by the DEC, but shows a violation for the central portion of the warp bubble.

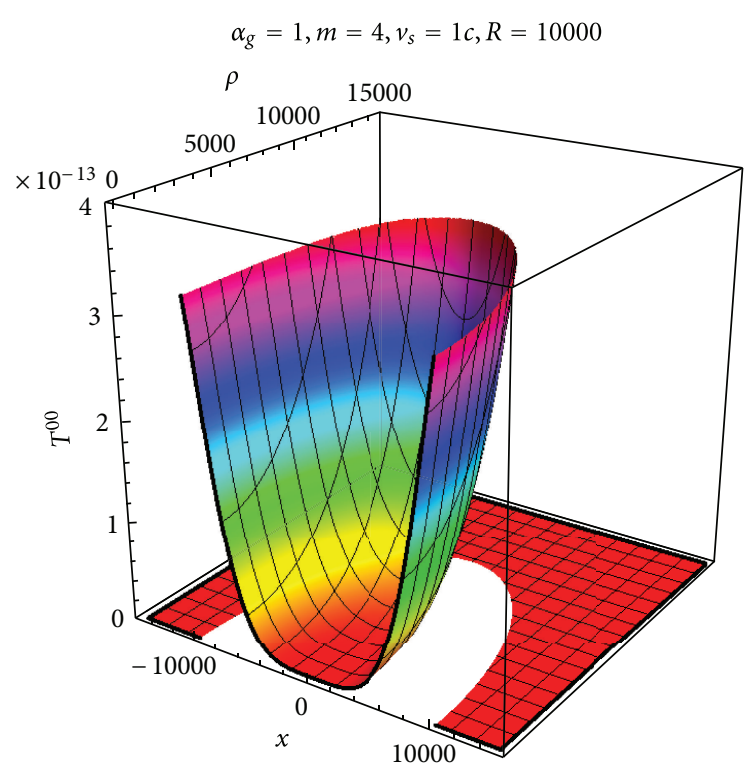

FIGURE 6: Energy density $T^{00}$ computed with conformal gravity and the Hartle shaping function, plotted for $\rho \geq 0$. Parameters used are $v_{s}=1, R=10000, m=4, \alpha_{g}=1$, and $t=0$. Integrating this local energy density over all space, we obtain an estimate for the total energy $E$ required to establish the warp drive.

value of $\alpha_{g}$ is not well established, since the number in (17) represents only an estimate of the macroscopic value of this coupling constant. This does not need to be the same as the microscopic value associated with the fundamental theory, which could be reduced by a factor of $N$, where $N$ could be the number of occupied baryonic states in a galaxy
$\left(N \sim 10^{68}\right)$, or possibly the number of baryons in the universe $\left(N \sim 10^{80}\right)[41]$.

Therefore, our estimate could be reduced by many orders of magnitude. Moreover, the energy necessary to establish the warp drive might also be decreased by using a more efficient shaping function, an analysis which we leave for a future study on the subject.

\section{Conclusions}

In this paper, we have analyzed in detail the Alcubierre warp drive mechanism within the framework of conformal gravity. We have seen that a particular choice of the shaping function (Hartle shaping function, instead of the original Alcubierre one) can overcome the main limitation of the AWD in standard general relativity, namely, the violation of the weak energy condition.

In fact, we have shown that for a wide range of spaceship velocities, the CG solutions do not violate the WEC, and, therefore, the AWD mechanism might be viable, if CG is the correct extension of the current gravitational theories. All the components of the stress-energy tensor can be analytically calculated, using a Mathematica program based on conformal gravity. Thus, a warp drive can, at least in principle, be fully established following our computations.

We have also checked two other main energy conditions: the SEC is always verified, while the DEC is violated, at least in the case we considered. Finally, we estimated the energy needed to establish a reasonable warp drive at the speed of light. This energy depends critically on the value of $\alpha_{g}$, the CG coupling constant, which is not well known. Therefore, this estimate will need to be refined in future studies.

\section{Appendix}

\section{Energy Density Expression in Conformal Gravity}

We present here the expression for the energy density $T^{00}$ in conformal gravity, computed using our Mathematica program. This is the general form of $T^{00}$ for any shaping function $f\left[r_{s}\right]$ and its derivatives, up to the fourth order. The energy density is a function of coordinates $t, x, \rho=\sqrt{y^{2}+z^{2}}$, and, therefore, it has a cylindrical symmetry around the $x$ axis. The distance $r_{s}$ is defined in (11), $v_{s}$ is the spaceship velocity, and $\alpha_{g}$ is the conformal gravity coupling constant:

$$
\begin{aligned}
T^{00}=\alpha_{g} \frac{v_{s}^{2}}{3 r_{s}{ }^{6}} & \left(-4 r_{s} v_{s}^{2} \rho^{2}\left(6\left(x-v_{s} t\right)^{2}+5 \rho^{2}\right)\left(-1+f\left[r_{s}\right]\right)\right. \\
& \times f^{\prime}\left[r_{s}\right]^{3}+4 r_{s}^{4} v_{s}^{2}\left(\left(x-v_{s} t\right)^{2}+3 \rho^{2}\right) \\
& \times f^{\prime}\left[r_{s}\right]^{4}+f^{\prime}\left[r_{s}\right]^{2} \\
& \times\left(-24\left(x-v_{s} t\right)^{4}+3\left(1+5 v_{s}^{2}\right)\right. \\
& \times\left(x-v_{s} t\right)^{2} \rho^{2}+\left(27+10 v_{s}^{2}\right) \rho^{4}
\end{aligned}
$$




$$
\begin{aligned}
& +v_{s}^{2}\left(5 \rho^{2}\left(3\left(x-v_{s} t\right)^{2}+2 \rho^{2}\right)\right. \\
& \times\left(-2+f\left[r_{s}\right]\right) \\
& \times f\left[r_{s}\right]-4 r_{s}{ }^{2}\left(x-v_{s} t\right)^{2} \\
& \times\left(4\left(x-v_{s} t\right)^{2}+3 \rho^{2}\right) \\
& \left.\left.\times\left(-1+f\left[r_{s}\right]\right) f^{\prime \prime}\left[r_{s}\right]\right)\right) \\
& -2 r_{s} f^{\prime}\left[r_{s}\right]\left(16\left(x-v_{s} t\right)^{2}-8 \rho^{2}\right. \\
& +\left(-60\left(x-v_{s} t\right)^{4}\right. \\
& +\left(-97+5 v_{s}^{2}\right)\left(x-v_{s} t\right)^{2} \rho^{2} \\
& \left.+\left(-37+3 v_{s}^{2}\right) \rho^{4}\right) f^{\prime \prime}\left[r_{s}\right] \\
& +r_{s}\left(4\left(-4+v_{s}^{2}\right)\right. \\
& \times\left(x-v_{s} t\right)^{4}+3\left(-9+v_{s}^{2}\right) \\
& \left.\times\left(x-v_{s} t\right)^{2} \rho^{2}-11 \rho^{4}\right) \\
& \times f^{(3)}\left[r_{s}\right]+v_{s}^{2} f\left[r_{s}\right]^{2} \\
& \times\left(\rho^{2}\left(5\left(x-v_{s} t\right)^{2}+3 \rho^{2}\right)\right. \\
& \times f^{\prime \prime}\left[r_{s}\right]+r_{s}\left(x-v_{s} t\right)^{2} \\
& \times\left(4\left(x-v_{s} t\right)^{2}+3 \rho^{2}\right) \\
& \left.\times f^{(3)}\left[r_{s}\right]\right)+2 f\left[r_{s}\right] \\
& \times\left(-8\left(x-v_{s} t\right)^{2}+4 \rho^{2}+v_{s}^{2}\right. \\
& \times\left(-\rho^{2}\left(5\left(x-v_{s} t\right)^{2}+3 \rho^{2}\right)\right. \\
& \times f^{\prime \prime}\left[r_{s}\right]-r_{s}\left(x-v_{s} t\right)^{2} \\
& \times\left(4\left(x-v_{s} t\right)^{2}+3 \rho^{2}\right) \\
& \left.\left.\left.\times f^{(3)}\left[r_{s}\right]\right)\right)\right) \\
& +r_{s}{ }^{2}\left(-16\left(2\left(x-v_{s} t\right)^{2}-\rho^{2}\right)\right. \\
& \times\left(-1+f\left[r_{s}\right]\right) f^{\prime \prime}\left[r_{s}\right] \\
& +\left(4\left(6+v_{s}^{2}\right)\left(x-v_{s} t\right)^{4}\right. \\
& +\left(43+3 v_{s}^{2}\right)\left(x-v_{s} t\right)^{2} \rho^{2} \\
& +19 \rho^{4}+v_{s}^{2}\left(x-v_{s} t\right)^{2} \\
& \times\left(4\left(x-v_{s} t\right)^{2}+3 \rho^{2}\right) \\
& \left.\times\left(-2+f\left[r_{s}\right]\right) f\left[r_{s}\right]\right)
\end{aligned}
$$

$$
\begin{gathered}
\times f^{\prime \prime}\left[r_{s}\right]^{2}+8 r_{s}\left(-1+f\left[r_{s}\right]\right) \\
\times\left(\left(2\left(x-v_{s} t\right)^{2}+\rho^{2}\right) f^{(3)}\left[r_{s}\right]\right. \\
\left.\left.\left.\quad+r_{s}\left(x-v_{s} t\right)^{2} f^{(4)}\left[r_{s}\right]\right)\right)\right) .
\end{gathered}
$$

In the main part of our work, we used the Hartle shaping function in (13), or more explicitly as

$$
f\left[r_{s}\right]=\left[1-\left(\frac{r_{s}}{R}\right)^{m}\right]^{+} \equiv \frac{\left|1-\left(r_{s} / R\right)^{m}\right|+\left[1-\left(r_{s} / R\right)^{m}\right]}{2}
$$

where $[\cdots]^{+}$indicates the positive part of the function. The derivatives of the HSF, up to the fourth order, were computed in terms of the Heaviside step function $H(x)$ and the Dirac delta function $\delta(x)$, also using the relations for the derivatives of these special functions $d|x| / d x=\operatorname{sgn}(x)=2 H(x)-1$; $d H(x) / d x=\delta(x) ; x^{n} d^{n} \delta(x) / d x^{n}=(-1)^{n} n ! \delta(x)$ as follows:

$$
\begin{aligned}
& f^{\prime}\left[r_{s}\right]=-\frac{m}{R}\left(\frac{r_{s}}{R}\right)^{m-1} H\left[1-\left(\frac{r_{s}}{R}\right)^{m}\right] \\
& f^{\prime \prime}\left[r_{s}\right]=-\frac{m(m-1)}{R^{2}}\left(\frac{r_{s}}{R}\right)^{m-2} H\left[1-\left(\frac{r_{s}}{R}\right)^{m}\right] \\
& +\frac{m^{2}}{R^{2}}\left(\frac{r_{s}}{R}\right)^{2 m-2} \delta\left[1-\left(\frac{r_{s}}{R}\right)^{m}\right] \\
& f^{(3)}\left[r_{s}\right]=-\frac{m(m-1)(m-2)}{R^{3}}\left(\frac{r_{s}}{R}\right)^{m-3} H\left[1-\left(\frac{r_{s}}{R}\right)^{m}\right] \\
& +\frac{3 m^{2}(m-1)}{R^{3}}\left(\frac{r_{s}}{R}\right)^{2 m-3} \delta\left[1-\left(\frac{r_{s}}{R}\right)^{m}\right] \\
& +\frac{m^{3}}{R^{3}}\left(\frac{r_{s}}{R}\right)^{3 m-3} \frac{\delta\left[1-\left(r_{s} / R\right)^{m}\right]}{\left[1-\left(r_{s} / R\right)^{m}\right]} \\
& f^{(4)}\left[r_{s}\right]=-\frac{m(m-1)(m-2)(m-3)}{R^{4}}\left(\frac{r_{s}}{R}\right)^{m-4} \\
& \times H\left[1-\left(\frac{r_{s}}{R}\right)^{m}\right] \\
& +\frac{m^{2}(m-1)(7 m-11)}{R^{4}}\left(\frac{r_{s}}{R}\right)^{2 m-4} \\
& \times \delta\left[1-\left(\frac{r_{s}}{R}\right)^{m}\right] \\
& +\frac{6 m^{3}(m-1)}{R^{4}}\left(\frac{r_{s}}{R}\right)^{3 m-4} \frac{\delta\left[1-\left(r_{s} / R\right)^{m}\right]}{\left[1-\left(r_{s} / R\right)^{m}\right]} \\
& +\frac{2 m^{4}}{R^{4}}\left(\frac{r_{s}}{R}\right)^{4 m-4} \frac{\delta\left[1-\left(r_{s} / R\right)^{m}\right]}{\left[1-\left(r_{s} / R\right)^{m}\right]^{2}} .
\end{aligned}
$$


By inserting these derivatives into (A.1), we obtained the expression used to compute $T^{00}$ in Figure 1 (bottom right panel) and Figures 2, 4, and 6.

\section{Acknowledgment}

This work was supported by a grant from the Frank R. Seaver College of Science and Engineering, Loyola Marymount University. The authors would like to acknowledge suggestions and clarifications by Dr. P. Mannheim and also thank the anonymous reviewers for the useful comments received.

\section{References}

[1] M. Alcubierre, "The warp drive: hyper-fast travel within general relativity," Classical and Quantum Gravity, vol. 11, p. L73, 1994.

[2] S. Hawking and G. Ellis, The Large Scale Structure of Space-Time, Cambridge University Press, New York, NY, USA, 1973.

[3] S. M. Carroll, Spacetime and Geometry: An Introduction to General Relativity, Addison-Wesley, San Francisco, Calif, USA, 2004.

[4] R. M. Wald, General Relativity, The University of Chicago Press, Chicago, Ill, USA, 1984.

[5] S. Krasnikov, "Hyperfast travel in general relativity," Physical Review D, vol. 57, pp. 4760-4766, 1998.

[6] J. Natario, "Warp drive with zero expansion," Classical and Quantum Gravity, vol. 19, no. 6, p. 1157, 2002.

[7] K. D. Olum, "Superluminal travel requires negative energies," Physical Review Letters, vol. 81, pp. 3567-3570, 1998.

[8] C. van den Broeck, "A "warp drive" with more reasonable total energy requirements," Classical and Quantum Gravity, vol. 16, no. 12, p. 3973, 1999.

[9] C. Clark, W. A. Hiscock, and S. L. Larson, "Null geodesics in the Alcubierre warp-drive spacetime: the view from the bridge," Classical and Quantum Gravity, vol. 16, no. 12, p. 3965, 1999.

[10] P. F. Gonzalez-Diaz, “ On the warp drive space-time," Physical Review D, vol. 62, Article ID 044005, 7 pages, 2000.

[11] M. J. Pfenning and L. Ford, "The unphysical nature of 'warp drive," Classical and Quantum Gravity, vol. 14, no. 17, p. 1743, 1997.

[12] H. G. White, "A discussion of space-time metric engineering," General Relativity and Gravitation, vol. 35, no. 11, pp. 2025-2033, 2003.

[13] F. S. Lobo and M. Visser, "Fundamental limitations on "warp drive" spacetimes," Classical and Quantum Gravity, vol. 21, p. 5871, 2004.

[14] P. F. Gonzalez-Diaz, "Superluminal warp drive," Physics Letters $B$, vol. 653, pp. 129-133, 2007.

[15] P. F. Gonzalez-Diaz, "Superluminal warp drive and dark energy," Physics Letters B, vol. 657, pp. 15-19, 2007.

[16] S. Finazzi, S. Liberati, and C. Barcelo, "Semiclassical instability of dynamical warp drives," Physical Review D, vol. 79, Article ID 124017, 2009.

[17] C. Barcelo, S. Finazzi, and S. Liberati, "On the impossibility of superluminal travel: the warp drive lesson," http://arxiv .org/abs/1001.4960. In press.

[18] T. Muller and D. Weiskopf, "Detailed study of null and time-like geodesics in the Alcubierre Warp spacetime," General Relativity and Gravitation, vol. 44, p. 509, 2012.
[19] B. McMonigal, G. F. Lewis, and P. O’Byrne, “The alcubierre warp drive: on the matter of matter," Physical Review D, vol. 85, p. 064024, 2012.

[20] J. B. Hartle, Gravity: An Introduction to Einstein General Relativity, Addison-Wesley, San Francisco, Calif, USA, 2003.

[21] P. D. Mannheim, "Alternatives to dark matter and dark energy," Progress in Particle and Nuclear Physics, vol. 56, pp. 340-445, 2006.

[22] G. U. Varieschi, "A kinematical approach to conformal cosmology," General Relativity and Gravitation, vol. 42, pp. 929-974, 2010.

[23] H. Weyl, "Reine infinitesimalgeometrie," Mathematische Zeitschrift, vol. 2, no. 3-4, pp. 384-411, 1918.

[24] H. Weyl, "Gravitation und elektrizität," Sitzungsberichte der Preussischen Akademie der Wissenschaften, Physikalisch-Mathematische, vol. 1918, p. 465, 1918.

[25] H. Weyl, "Eine neue Erweiterung der Relativit tstheorie," Annalen der Physik, vol. 59, pp. 101-133, 1919.

[26] P. D. Mannheim, "Making the case for conformal gravity," Foundations of Physics, vol. 42, p. 388, 2012.

[27] P. D. Mannheim and J. G. O'Brien, "Impact of a global quadratic potential on galactic rotation curves," Physical Review Letters, vol. 106, no. 12, Article ID 121101, 2011.

[28] P. D. Mannheim and J. G. O'Brien, "Fitting galactic rotation curves with conformal gravity and a global quadratic potential," Physical Review D, vol. 85, no. 12, Article ID 124020, 26 pages, 2012.

[29] J. G. O'Brien and P. D. Mannheim, "Fitting dwarf galaxy rotation curves with conformal gravity," Monthly Notices of the Royal Astronomical Society, vol. 421, no. 2, pp. 1273-1282, 2011.

[30] P. D. Mannheim, "Schwarzschild limit of conformal gravity in the presence of macroscopic scalar fields," Physical Review D, vol. 75, Article ID 124006, 2007.

[31] G. U. Varieschi, "Kinematical conformal cosmology: fundamental parameters from astrophysical observations," ISRN Astronomy and Astrophysic, vol. 2011, Article ID 806549, 24 pages, 2011.

[32] A. Diaferio, L. Ostorero, and V. F. Cardone, "Gamma-ray bursts as cosmological probes: lambdaCDM versus conformal gravity," Journal of Cosmology and Astroparticle Physics, vol. 2011, Article ID 008, 2011.

[33] S. Weinberg, Gravitation and Cosmology: Principles and Applications of the General Theoryof Relativity, John Wiley \& Sons, New York, NY, USA, 1972.

[34] R. Bach, "Zur Weylschen Relativittstheorie und der Weylschen Erweiterung des Krummungstensorbegriffs," Mathematische Zeitschrift, vol. 9, pp. 110-135, 1921.

[35] C. Lanczos, "A remarkable property of the riemann-christoffel tensor in four dimensions," Annals of Mathematics, vol. 39, pp. 842-850, 1938.

[36] R. Schimming and H. J. Schmidt, "On the history of fourth order metric theories of gravitation," NTM Schriftenreihe für Geschichte der Naturwissenschaften, Technik und Medizin, vol. 27, p. 41, 1990.

[37] P. D. Mannheim and D. Kazanas, "Exact vacuum solution to conformal Weyl gravity and galactic rotation curves," The Astrophysical Journal, vol. 342, pp. 635-638, 1989.

[38] D. Kazanas and P. D. Mannheim, "General structure of the gravitational equations of motion in conformal Weyl gravity," The Astrophysical Journal, vol. 76, p. 431, 1991. 
[39] H. J. Schmidt, "Non-trivial solutions of the bach equation exist," Annalen der Physik, vol. 496, no. 6, pp. 435-436, 1984.

[40] J. Wood and W. Moreau, "Solutions of conformal gravity with dynamicalmass generation in the solar system," http://arxiv.org/ abs/gr-qc/0102056. In press.

[41] P. Mannheim, private communication,2012. 

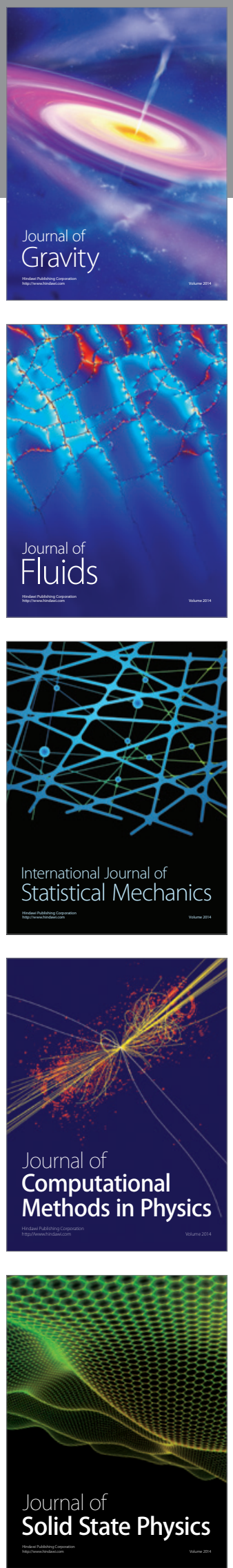

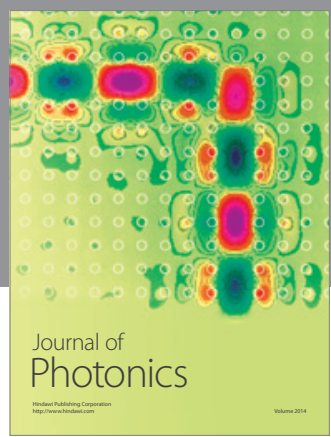

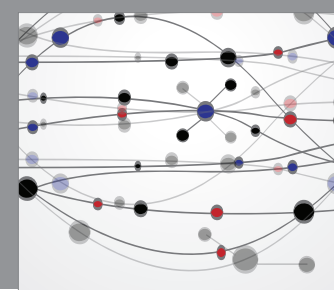

The Scientific World Journal

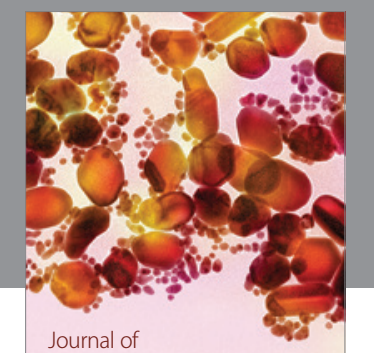

Soft Matter
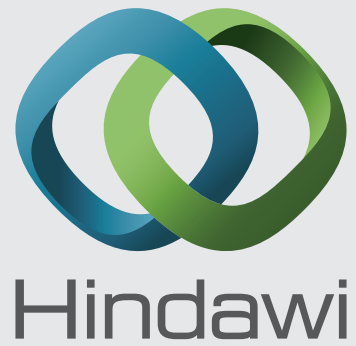

Submit your manuscripts at

http://www.hindawi.com
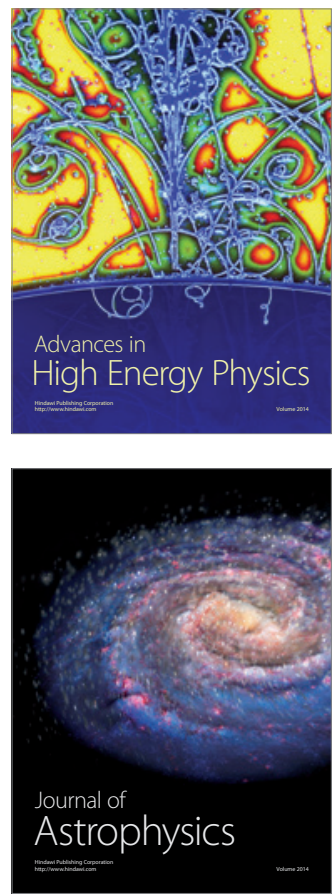
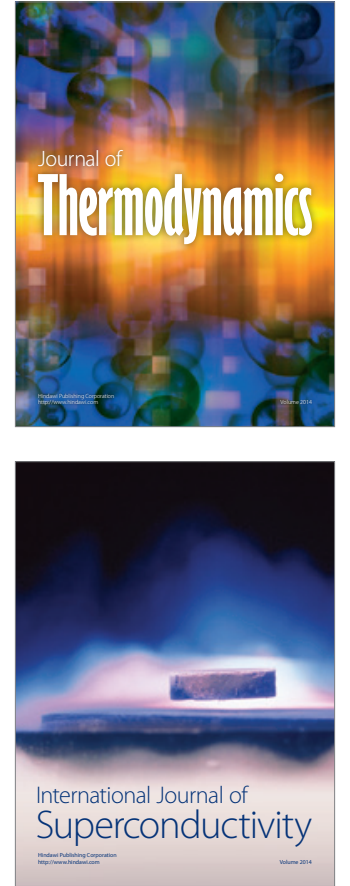
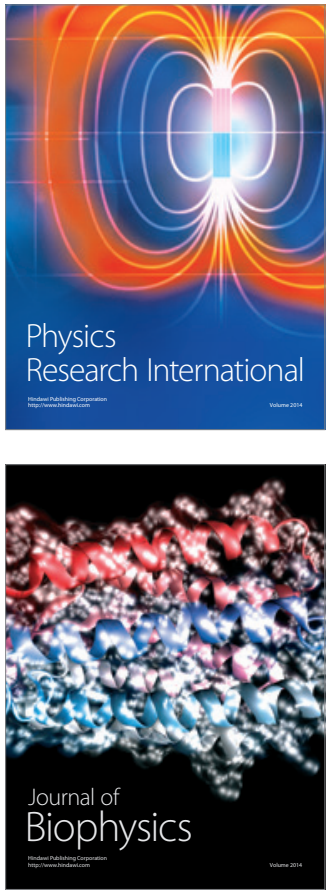
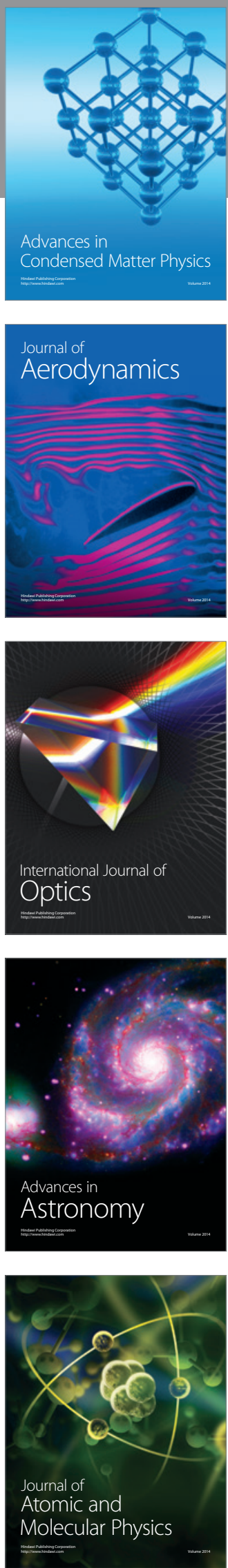